\title{
Experiences of Caretakers Seeking Care for Their Under-five Children Related to Public Primary Health Care in Sharg-Alneel Locality, 2015
}

\author{
Malaz Elbashir Ahmed ${ }^{1, *}$, Khalid Fadl Alla Khalid ${ }^{2}$, Talal Elfadil Mahi ${ }^{3}$, Nada Jafar Osman ${ }^{4}$, \\ Ibrahim Ismail Mohammed Abu ${ }^{5,6}$, Nada Mohamed Ali Alnair ${ }^{7}$, Rania Hassan Abdelgfour ${ }^{2}$ \\ ${ }^{1}$ Free Lance Community Medicine Consultant, Khartoum, Sudan \\ ${ }^{2}$ HIV Prevention Program, United Nations Population Fund, Khartoum, Sudan \\ ${ }^{3}$ Community Medicine, National Health Insurance Fund, Khartoum, Sudan \\ ${ }^{4}$ Community Medicine, Primary Health Care Directorate, Federal Ministry of Health, Khartoum, Sudan \\ ${ }^{5}$ Community Medicine Department, Faculty of Medicine, Al-Fashir University, Al-Fashir, Sudan \\ ${ }^{6}$ Community Medicine Department, Faculty of Medicine, King Abdulaziz University, Jeddah, Saudi Arabia \\ ${ }^{7}$ Continuous Professional Development Directorate, Federal Ministry of Health, Khartoum, Sudan
}

\section{Email address:}

malazelbashir@gmail.com (M. E. Ahmed),elmalkey2011@gmail.com (K. H. A. Khalid), talalelfadil@yahoo.com (T. E. Mahi), nadajafar@gmail.com (N. J. Osman), burha75@gmail.com (I. M. Abu), nadaalnayer@gmail.com (N. M. Alnair), drraniahassan@gmail.com (R. H. Abdelgafour)

${ }^{*}$ Corresponding author

\section{To cite this article:}

Malaz Elbashir Ahmed, Khalid Fadl Alla Khalid, Talal Elfadil Mahi, Nada Jafar Osman, Ibrahim Ismail Mohammed Abu, Nada Mohamed Ali Alnair, Rania Hassan Abdelgfour. Experiences of Caretakers Seeking Care for Their Under-five Children Related to Public Primary Health Care in Sharg-Alneel Locality, 2015. Science Journal of Public Health. Vol. 7, No. 2, 2019, pp. 59-65.

doi: $10.11648 /$ j.sjph.20190702.15

Received: September 24, 2018; Accepted: April 8, 2019; Published: May 27, 2019

\begin{abstract}
Sudan public sectors' has three levels of services delivery: primary, secondary and tertiary care. Primary level intended for health facilities that provide the basic package of health services and supposed to be the first contact for seeking medical help, advices and referral to high specialized services. Therefore, coordination between these three levels is important. As well as bypassing primary care level results in overburdening of referral facilities, increasing cost for the patients and depreciating health care system in terms of manpower, equipment and resources. Information about the health services utilizations' and it's the associated factors will be useful for improving service delivery to achieve universal health coverage. This paper intended to explore the experiences of caretakers seeking care for their under-five children related in public PHC facilities in Sharg-Alneel Locality, 2015. The study was descriptive cross-sectional applied qualitative research methods. Focus group discussions were conducted with caretakers who had experience with PHC services. Qualitative data was manually analyzed using thematic content analysis. This paper revealed that the main reasons that force the respondents to bypass the primary health facilities and seek care directly from the hospitals were related to the quality of the provided services. Accessibility issues, affordability issues, lack of knowledge of existing services, inadequacy of drugs, health staff competency and proper referrals system which is crucial to a functioning PHC, also reported to be lacking. Also, in this paper the respondents recommend the expansion of health insurance services coverage, provision of ambulances to all PHC facilities. Therefore, this paper recommends to educate and sensitize the individuals, families and community on PHC services role as the first line of health services to increase their knowledge and create awareness of services provided at the PHC facilities. This will increase demand for and improve access to PHC services. Also, to strengthen the implementation and facilitate the monitoring and evaluation by the authorities at the different levels. To enhance role of health insurance fund as purchaser rather than services provider and breach of free health care policy jeopardized affordability of PHC services need urgent actions, and continuous professional development for the PHC healthcare providers which will contribute in improving performance and providing quality $\mathrm{PHC}$, and building their capacity on management of the under- 5 common illness and by
\end{abstract}


strengthen the supportive supervision and monitoring.

Keywords: Primary Health Care, Service Delivery Levels, Factors Associated with Bypassing Behaviour, Caretakers Experience, Adequacy of Services, Referral System, Quality Primary Health Care Services

\section{Introduction}

Sudan public health sectors' has three levels of services delivery: primary, secondary and tertiary care. Primary level are health facilities that provide the basic package of health care and supposed to be the first contact for seeking medical help, advices and referral to high specialized services. However, the coordination between these three levels of healthcare services is poor and hence the providers working at those facilities [1]. The health care system financing is $7 \%$ of gross domestic product (GDP) and 9\% of the budget of the government which is far below the target of $15 \%$ set in the Abuja Declaration [1]. Although the government allocation for health is very low, yet it is evidenced that $80 \%$ of these funds are spent in tertiary services [2].

The provision of PHC services in Sudan is arranged across four levels of services according to the population size. Each level is provided by specific type of facilities that has standardized service profile, staffing and supported by a standardized set of management and supportive components. The four levels of service are; community services; family health units; family health centers and local hospitals. In urban areas the FHCs are staffed by medical officer and paramedics in contrast to rural areas where they are staffed by paramedics [3- 4]. In 2008 the PHC services mapping showed that only $19 \%$ of primary health care (PHC) sites provided the PHC Minimum Package ( e.g. Integrated management of child hood illness (IMCI), vaccination, antenatal care and family planning, growth monitoring and nutrition education and provision of essential drugs), and the geographical accessibility to PHC sites is 1:7,000 [5- 6]. PHC is the best way to combat major causes of under-5 children mortality and hence achieving SDG goal-4.

According to Sudan referral system, PHC facilities are supposed to be the first contact for mild childhood illnesses. The key reasons for deciding to refer either an emergency or routine case include; seeking expert opinion, to seek additional or different services, to seek admission and management of the client, to seek use of diagnostic or therapeutic tools not available at initiating facility [7]. The referral system in Sudan is less formal (no gate keeping) with the Patients directly visit specialists without restrictions. In 2008 WHO reported that seven of the developed countries' systems feature primary care gate keeping; in the remainder, patients self-refer to specialists. Only New Zealand, the Netherlands and the United Kingdom require that patients formally enroll with a primary care physician [8-9-10]. In 2009, a referral system approach has been introduced in Khartoum state and all patients were obliged to visit their PHC provider first before receiving specialist care. The successful implementation of formalization of referral system in Khartoum state was evident from the reports submitted by the hospitals. Nevertheless, after the devolution of hospitals to the states in 2012 Referral system in Khartoum state became less Formalized [11]. In the same year (2012) Sudan introduced The Expansion of the PHC Services Initiative, with the objectives of improving the availability and achieving full population access to the essential package of PHC services, and ensuring the quality and sustainability of PHC services. In 2016 Elbashir indicated that almost 9 in 10 care takers interviewed had directly chosen the secondary level without referral despite extra time and cost. And among those who were referred only $30 \%$ their source of referral is public PHC facilities. The main reason given for directly choosing the hospitals is better quality of care.

Sudan has a high rate of mortality among children under age of 5 caused by diseases that can be simply prevented and treated at PHC facilities which are made easily accessible to the population [12] Although the number of under-five deaths worldwide has decreased by half since 1990, many countries including Sudan are not on track to reach Sustainable Development Goal 4 (SDG4), which calls for a two-thirds reduction in under-five mortality by 2015. Socioeconomic inequities' existing in many developing countries is one of the reasons for slow progress in achieving SDG-4. These injustices may jeopardize access to and utilization of available health facilities [13], time taken in seeking of health care as well as selection of appropriate health care provider for acute childhood illnesses [14-15].

Availability of the appropriate health workers with adequate resources is essential to provide the quality care to those who need it [16]. In Sri-Lanka all characteristics of providers are found to have the theoretically expected relationships to facility choice [11]. Another provider reason for bypassing PHC facilities given by caretakers in quantitative and qualitative studies from Tanzania was lack of qualified personnel at such facilities [17, 18]. Causes of underuse of Primary Care in China are multiple and include major historic and institutional factors such as severe maldistribution of human resources and lack of primary care practitioners, lack of a functional gate-keeping mechanism and the low educational attainment of health care providers [19]. Good quality care includes proper and effective treatment and referrals and ready access to necessary medicines and medical supplies [15]. In Sri-Lanka all characteristics of facilities are found to have the theoretically expected relationships to facility choice. Higher prices tend to deter use of the facility, while improved quality of services increases the likelihood of that facility being chosen [11]. In Kenya the main reasons cited for by-pass were poor care $(21 \%)$, lack of drugs and supplies (17\%) and lack of poor laboratory services $(12 \% /)$ [20]. From the facility audit, the 
worst areas were in availability of drugs, equipment and management issues. Visitors to community health institutions in China were found to have lower satisfactions in terms of medical equipment and drug prices [21]. The main reasons given for bypassing the PHC facilities in quantitative and qualitative studies conducted in rural Tanzania were the lack of diagnostic facilities, lack of equipment to test for malaria and blood hemoglobin level, lack of drugs [17, 18]. One of the main findings of cross- sectional interview surveys in Papua New Guinea related to choosing healthcare providers for febrile children that drug availability is significantly increased the probability of Village Health Volunteers utilization [22]. Study conducted in Ethiopia revealed that; obtaining laboratory test, availability of drugs at closer health facility, were significantly associated with self-referral to General Hospital [23].

Sudan is committed to provide affordable and universal health coverage and has started the PHC expansion project. However, the expansion project in Sudan have been burdened by many challenges including financing issues, accessibility to medical products and technologies, shortage and poor capacity of the workforce, inadequacy of health information systems, and governance. Furthermore, a few studies were conducted about utilization of health services in Sudan, with no published country level reports about type of health services utilized for childhood illnesses. The un-published routine data is indicating that the referral system is inadequately performing at all the seven localities in Khartoum state. This research is intended to explore the experiences of caretakers seeking care for their under-five children related to public PHC facilities in Sharg-Alneel Locality.

\section{Materials and Methods}

\subsection{Study Design}

This was descriptive cross-sectional paper applied qualitative research method.

\subsection{Study Area}

Sharg-Alneel locality was selected as study area for this study because it has a high reported cases of childhood illnesses, wide range of PHC facilities and its Priority locality for Federal Ministry of Health.

\subsection{Study Population}

The study participants were that Mothers, fathers or other caretakers with children who are between one month to five years and had experiences in requesting the primary health care service in Sharg-Alneel locality. In this study the caretakers who are live outside Sharg-Alneel locality were excluded.

\subsection{Sample Size and Sampling Method}

The purposive sampling method applied for caretakers of under-five children, and hence 66 caretakers who had an experiences in requesting the primary health service and living in the catchment area of the 4 hospitals were selected, i.e. 32 women and 34 men were participated in this study.

\subsection{Data Collection Tools}

Focus group discussion (FGD) with the open ended questions used to collect data from the caretakers of underfive children. The study participants organized in eight focus group discussions (FGDs) in four different geographical/PHC catchment areas at Sharg-Alneel locality with range of 6-12 participants per each. Four groups of male's caretakers and 4 groups of female's caretakers were conducted in period between February and March 2016 in Abo delag, Wad abo salih, Alban jaded, and Om dawan ban.

\subsection{Data Collection Technique}

(a) A guide was used to introduce topics for discussion in the form of open ended questions.

(b) The discussions were held in closed halls within the hospitals and lasted in 45 to 60 minutes.

(c) The discussions were conducted in Arabic and led by two data collectors

(d) Received information by respondents was noted and tape recorded.

(e) After that the noted and audio materials were transcribed, coded, categorized and then grouped under meaningful themes.

(f) Initial word to word transcription of the audio materials was done by one of the research assistants, and at last the translation into English was done by the principal investigator.

\subsection{Quality Assurance}

Data cleaned manually by examining the collected raw data during which errors were detected and corrected by omission.

\subsection{Data Analysis}

The data was analyzed thematically by frame work analysis. Firstly data analysis matrix with labels related to the issues researched were developed. Then the transcript data entered and adapted by making a brief note (familiarization). Accordingly, the researched issues were summarized and the findings described.

\subsection{Ethical Clearance}

Ethical clearance was obtained at the national level from the Sudan medical specialization board (SMSB) and Khartoum State ministry of health. In addition, permission to perform the study obtained from the different hospital directors. Verbal consent was obtained from the respondents after an explanation of the interview aims. Participation was on a completely free will basis. All approached respondents agreed to participate in the study. 


\section{Results}

The results showed that $49 \%$ of participants were female. The ratio of male to female participants almost equal [Male to Female equal [32 female and 34 male]. The collected findings were categorized into six themes; i.e. health care seeking behavior during child illnesses, availability of health care facilities, health facility choice preference during child illness, Quality of public PHC facilities, magnitude of and reasons behind bypassing behavior, role of community health workers, obstacles faced by caretakers while seeking care for their sick under five children at the public PHC facilities in their area, and suggestions to improve PHC services.

Table 1. Composition of FDGs.

\begin{tabular}{llll}
\hline \multirow{2}{*}{ Catchment areas } & \multirow{2}{*}{ FGDs } & \multicolumn{2}{l}{ Participants } \\
\cline { 3 - 4 } & & Men & Women \\
\hline Abo delag & 2 & 12 & 10 \\
Wad abo salih & 2 & 10 & 9 \\
Alban jaded & 2 & 6 & 6 \\
Om dawan ban & 2 & 6 & 7 \\
Total & 8 & 34 & 32 \\
\hline
\end{tabular}

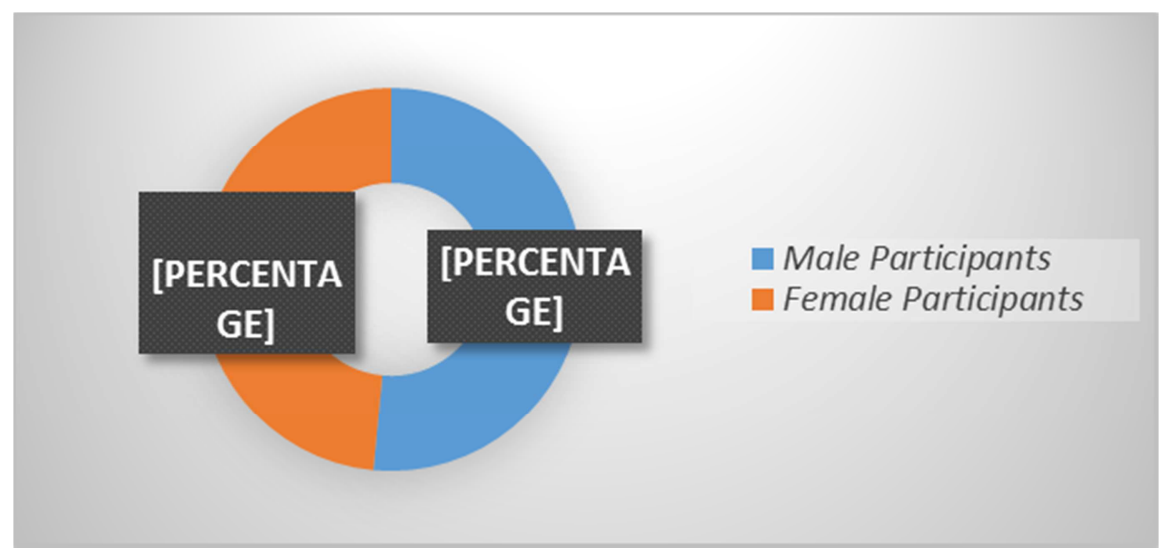

Figure 1. Percentage of male to female respondents.

Health care seeking behavior during child illnesses: The majority of participants mentioned that "we go to the hospital directly for care". While a minority of them mentioned "initially we go to nearby primary health facilities, and if the child does not improve then we go to the hospital". A minority of respondents also said that "we go to the Sheikh first to get cured using (mahaya and Albkhra) and then go to the hospital if we have not got better or if referred to by Sheikh". Majority of the participants also noted that "there were no primary health care facilities near our neighborhoods and that the hospital is the closest facility for us". Few of them also pointed to the existence of health centers and a minority of them pointed to the existence family health units.

Health facility choice preference during child illness and Quality of public PHC facilities: All caretakers expressed their disappointments regarding quality of care provided at PHC facilities. Unpredictable availability of qualified health workers at PHC facilities was one of the issues raised across all the eight FGDs. Some participants said that "the care providers are not reachable at all times at the nearby health institutions". The same concern was raised by caretakers from a different area and noted that "existing staff at the nearer health facilities are not qualified so do not trust them in the treatment of our children". Some also pointed out that "the medical assistants became too old and unable to treat our children's".

Although family health centers and even family health unit are supposed to provide basic diagnostic services, such services were reported to be almost never available. The participants expressed their demand for such services "The proportion of laboratory services provided at the hospital higher than proportion provided at other health institutions so they go to the hospital immediately for seeking care". Some also mentioned lack of life saving measures "no oxygen at nearby health institutions to our homes", forcing them to go to the hospital immediately

Frequent shortage of drugs was reported to be commonly encountered at PHC facilities. One caretaker said "Provision of low cost drugs in the hospital compared with lack of and high cost drugs in the PHC facilities". Other caretaker reported that majority of care providers are working also as drug sellers.

Social Health Insurance (SHI) in Sudan fully implemented since 1995 yet the population coverage by SHI is still unsatisfactory. It is at $37.7 \%$ of the total populations and is mostly restricted to the formal sector. Many caretakers noted "No health insurance services in the nearer facilities compared to hospitals".

Public PHC facilities in Sudan operate between 8 am and 4 pm and are closed on weekends and public holidays. However, it was reported that sometimes the PHC facilities were closed even during the week days when no health staff was available to provide services. One of the caretakers said "public PHC facilities have restricted opening hours while the hospital operate 24 hours that is why If my child gets sick at night and can afford transport will go directly to the hospital".

According to Sudan health policy, health care services to children below five years is supposed to be provided free of charge at public health facilities. However, caretakers 
claimed to incur some costs for various services when they brought their children to the primary care facilities. This was raised by caretakers across all FGDs. The quotes below illustrate "High prices of public primary health care services like doctor consultation, investigations and drugs". Financial Inaccessibility of services resulting from the inability to pay was also mentioned by some of the respondents "Most of the citizens have low income and unable to afford the cost of services at PHC facilities so they preferred free services provided in the hospital".

Magnitude of and reasons behind bypassing behavior: According to Sudan referral system, PHC facilities are supposed to be the first contact for mild childhood illnesses and act as 'gatekeepers' with responsibility for defining which patients require secondary care. However, all participants; women and men; agreed that they are getting the best help in the hospital and therefore prefer to go to the hospital directly. And all reported the quality issues "opening hours, availability of qualified care providers, lack of trust on the care providers due to previous experience, availability of lab investigations/services, availability of drugs, high cost services and drugs" are the main reasons that force them to bypass public PHC and seek care directly from the hospitals.

Role of community health workers: All the study participants agreed that there are no community health workers in their areas. And some of them mentioned that they are not aware about care providers called community health workers.

Obstacles faced by caretakers while seeking care for their sick under-five children at the public PHC facilities in their areas: Beside all the quality issues mentioned earlier another thing complained about by caretakers was the lack of proper referral services when their children were referred to a higher level hospital. Across all the focus group discussions, caretakers complained about lack of transportation for referral of sick children from the PHC facilities to the first referral hospital as well as bureaucracy in the procedures of utilizing the available ambulances. One of the caretakers quoted that "ambulance cannot move unless permission received from the central ambulance and the doctor has no authority to order the ambulance to move and we have one child girl died due to this bureaucratic procedures".

Their suggestions to improve PHC services: At the end of the focus group discussions caretakers suggest some interventions they perceive that can help in improving PHC services, strengthening referral system and ultimately reduce bypassing PHC services for common child illnesses. Caretakers propose "Establishment of new quality PHC facilities, strengthening of available PHC facilities, and provision of outreach health care services". Also respondents recommend "Expansion of health insurance services coverage, provision of ambulances to all PHC facilities, and training of health workers from the area".

\section{Discussion}

The main objective of Sudan primary health care (PHC) reform, 2012-2016 is to increase population access to PHC services from $86 \%$ to $100 \%$ with the availability of free health care services policy for under- 5 children. However, the real use of the service has been low, extremely restricting the overall effectiveness of the health system response. This paper explore the experiences of caretakers seeking care for their under-five children related to public PHC facilities and the factors influencing bypassing PHC services for common child hood illnesses in Sharg-Alneel Locality from caretaker's point of view. PHC facilities are supposed to be the first contact for mild childhood illnesses and act as 'gatekeepers' with responsibility for defining which patients require secondary care.

This qualitative study was conducted among caretakers (mothers and fathers) who have children less than 5 years and have had previous experiences in requesting health care from PHC facility. Findings showed that majority of caretakers were bypassing PHC services for common child illnesses and sought care directly from secondary care level. All reasons that lead to bypassing behavior have been discussed with the caretakers were found to be related to quality of care, access and satisfaction.

Public PHC facilities in Sudan operate between 8 am and 4 pm and are closed on weekends and public holidays. Caretakers reported that Primary health care services cannot be accessed not 24 hours a day or during weekends and holidays.

Focus group discussions also resulted in suffering of caretakers from geographical inaccessibility of PHC services. Moreover, available services were characterized by shortage of drugs, health workers and a life saving measures and specifically oxygen was mentioned. Although PHC are supposed to provide basic diagnostic services, such services were reported to be almost never available, and the participants expressed their demand for such services.

Children below five years are exempted from the official user fees under the Free Care policy of Sudan announced by the President 2008 [24]. However, caretakers in this study notified paying for consultations, investigations and drugs. The issue of unofficial payments is disturbing; unacceptable and indicates poor monitoring of PHC services. This finding; has been also reported in Tanzania [18, 25]. Moreover, participants in this study reported the lack of health insurance services in primary health care facilities which confirms informal payment practice at PHC facilities. This finding is in line with the study assessed individual's perception of the quality of health services, and indicated the cost of service was a primary barrier to seek healthcare in Egypt.

Age and gender of the provider are crucial for promote satisfaction and acceptability of health services [26]. Several concerns were raised by caretakers in this study regarding the age of medical assistant; main service provider at PHC facilities. Finding, has been reported also by Khalid. etal. In his paper showed that majority of the care providers at primary health care service points were not trained and they lack the specific capacity to provide the service [27]

Referral is a process in which health workers at a lowest 
level of the health system seeks the assistance of a better resourced facility at the same or higher level [28]. Referral is a critical part of appropriate primary care and the Integrated Management of Childhood Illness (IMCI) strategy. Majority of caretakers interviewed pointed to the inadequately designed and poorly performed referral system.

The figures showed that only $55 \%$ were satisfied with the medical care they received, $57 \%$ said that the cost of service was problem and $70 \%$ complained about the long waiting time. In the face of the fact of the large number of free provided services by the ministry of health, yet $41 \%$ of $\mathrm{MOH}$ facility users want to switch, where only $15 \%$ of private user will do so. In both cases they will switch to another private facility [29]. Service quality by itself without considering its marketability does not produce differentiation. Thus the healthcare facilities should put a marketing mix strategy that customers perceive as good quality. The healthcare providers can influence the patient's perception of service received, by providing quality healthcare service in a patient focused environment. The patient then will easily communication his experience to family and friends [30]. Age and gender of the provider are crucial for promote satisfaction and acceptability of health services [31].

All determinants of bypassing behavior reported are mainly quality dimensions; availability accessibility, adequacy, acceptability and affordability of services. The results showed that either PHC services not available or study respondents were not aware about the existing services or they perceive that the hospital is nearer. If this indicates anything shows inequitable distribution of health services and discrepancy between services distribution and referral policy. Also, the reported the poor quality of PHC services indicating weak PHC services monitoring and control.

\section{Conclusion}

This is one of the first researches to explore the experiences of caretakers seeking care for their under-five children related to public PHC facilities in Sharg-Alneel Locality in Sudan. This study has shed light on the experiences that prevent caretakers from using closer PHC services and directly choose secondary hospital. In this study the main reasons given for bypassing the nearer PHC services were unavailability of doctors as well as high cost of services. According to Sudan health policy, health care services to children below five years is supposed to be provided free of charge at public health facilities. The qualitative findings pointed out important limitations in PHC services provision. Availability of PHC services, lack of knowledge of existing services, inadequacy of drugs and health staff impede the quality of PHC services. Breach of free health care policy jeopardized affordability of PHC services. Proper referrals system which is crucial to a functioning PHC, were also reported to be lacking. Therefore, educate and sensitize the individuals, families and community on PHC services role as the first line of health services to increase their knowledge and create awareness of services provided at the PHC facilities. This will increase demand for and improve access to PHC services. Also, to strengthen the implementation and facilitate the monitoring and evaluation by the authorities at the different levels. To enhance role of health insurance fund as purchaser rather than services provider, and continuous professional development for the PHC healthcare providers which will contribute in improving performance and providing quality PHC, and building their capacity on management of the under-5 common illness and by strengthen the supportive supervision and monitoring.

\section{References}

[1] FMOH: National Health Sector Strategic Plan 2012-2016. Khartoum: Policy and Planning Unit 2011.

[2] FMOH: Primary Health Care Revitalization Strategy. Khartoum: Directorate General for PHC and Preventive Medicine 2009.

[3] FMOH: National Human Resources for Health Strategic Plan for Sudan. Khartoum: Directorate General of Human Resources for Health Development 2011.

[4] FMOH: Specifications and standards for the health system in Sudan, Part II: Document of Characterization of services and health units. Khartoum: Policy and Planning Unit 2010.

[5] FMOH: Promoting access to high quality primary health care services in Sudan (policy brief). Khartoum: Policy and Planning Unit 2011.

[6] FMOH: Knowledge, Attitudes and Practices (KAP) in the Sudanese communities for their health seeking behavior: Policy and Planning Unit 2011.

[7] WHO: Referral Systems - a summary of key processes to guide health services managers. Geneva: World Health Organization no date.

[8] Robin Gauld et al: The World Health Report 2008-Primary Healthcare: How Wide Is the Gap between Its Agenda and Implementation in 12 High-Income Health Systems?, HEALTHCARE POLICY 2012, 7 (3).

[9] Coulter A, Bradlow J: Effect ofNHSreforms on general practitioners' referral patterns. British Medical Journal 1993; 306:433.

[10] Grol R M, Faber G, Voerman J, Burgers and S. Leatherman: "Primary Care in the 21st Century: What Can Be Learned from the Netherlands?" Paper under review 2010.

[11] KSMOH: referral system, Khartoum state experience. Khartoum: Directorate General for curative Medicine 2014.

[12] WHO: Reduction of child mortality in the Eastern Mediterranean Region. Geneva: World Health Organization 2012.

[13] Deogaonkar M: Socio-economic inequality and its effect on health care delivery in India: Inequality and health care. Electronic Journal of Sociology 2004. 
[14] Herman E, Black RE, Wahba S, Khallaf N: Developing strategies to encourage appropriate care-seeking for children with acute respiratory infections: an example from Egypt. Int J Health Plann Manage 1994, 9: 235-243.

[15] Thind A: Analysis of health services use for respiratory illness in Indonesian children: implications for policy. J BiosocSci2005, 37: 129-142.

[16] FMOH: National Maternal and Child Health Strategic Plan 2012-2016. Khartoum: Directorate General for PHC and Preventive Medicine 2011

[17] Kahabuka et al.: Why caretakers bypass Primary Health Care facilities for child care - a case from rural Tanzania. BMC Health Services Research 2011, 11:315.

[18] Kahabuka et al.: Unfulfilled expectations to services offered at primary health care facilities: Experiences of caretakers of under-five children in rural Tanzania. BMC Health Services Research 2012, 12:158.

[19] Wu D, Lam TP. Underuse of Primary Care in China: The Scale, Causes, and Solutions. JABFM 2016, Vol. 29 No.

[20] Audo MO, Ferguson A, Njoroge PK: Quality of health care and its effects in the utilization of maternal and child health services in Kenya. East Afr Med J 2006, 82(11).

[21] Dong et al: Focus on vulnerable populations andpromoting equity in health service utilization -an analysis of visitorcharacteristics and service utilization of the Chinese community healthservice. BMC Public Health 2014, 14:503.

[22] Tsukahara T., The Choice of Healthcare Providers for Febrile Children after Introducing Non-professional Health Workers in a Malaria Endemic Area in Papua New Guinea. 2015; $3: 275$.

[23] Wolkite Olani Abdi, Waju Beyene Salgedo, Gebeyehu Tsega
Nebeb. Magnitude and Determinants of Self-Referral of Patients at a General Hospital, Western Ethiopia. Science Journal of Clinical Medicine 2015; 4:5, 86-92.

[24] FMOH: Free healthcare policy for under-fives and pregnant women in northern Sudan: findings of a review. Khartoum: directorate general of planning and international health 2011.

[25] Mæstad O, Mwisongo A, Institute CM: Informal payments and the quality of health care in Tanzania: results from qualitative research. Chr. Michelsen institute (CMI) 2007.

[26] Peters D H, Garg A, Bloom G, Walker DG, BriegerWR, Rahman M. H: Poverty and access to health care in developing countries. Annals Of The New York Academy Of Sciences 2008; 1136:8, 161-171.

[27] Khalid. F. K, Samia. Y. I. H, Nada. J. O, Malaz. E. A, Rania. H. A Factors Affecting Patient's Satisfaction with Provided Sexually Transmitted Infections Primary Health Care Service in El-Damazin Locality at Blue Nile State, Sudan 2015 - 2016 World Journal of Public Health. Vol. 3, No. 3, 2018, pp. 93-98. doi: $10.11648 /$ j.wjph.20180303.14

[28] WHO: Referral Systems - a summary of key processes to guide health services managers. Geneva: World Health Organization no date.

[29] Peter Berman, Fatma El Zanaty, Healthcare Utilization and expenditure in the ARE. Harved University for decision making project.

[30] Sadok. J. M. Marketing Patients the best marketing spokespeople. Hosp Mater Manage Q, Nov. 1990; 13 (2):pp. 18-21.

[31] Peters D H, Garg A, Bloom G, WalkerDG, Brieger WR, Rahman M. H: Poverty and access to health care in developing countries. Annals Of The New York Academy Of Sciences 2008; 1136:8, 161-171. 